\title{
Temporal Changes in the Rigidity Spectrum of Forbush Decreases Based on Neutron Monitor Data
}

\author{
M.V. Alania • A. Wawrzynczak · V.E. Sdobnov • \\ M.V. Kravtsova
}

Received: 7 October 2012 / Accepted: 6 March 2013 / Published online: 29 March 2013

(C) The Author(s) 2013. This article is published with open access at Springerlink.com

\begin{abstract}
The Forbush decrease (Fd) of the Galactic cosmic ray (GCR) intensity and disturbances in the Earth's magnetic field generally take place simultaneously and are caused by the same phenomenon, namely a coronal mass ejection (CME) or a shock wave created after violent processes in the solar atmosphere. The magnetic cut-off rigidity of the Earth's magnetic field changes because of the disturbances, leading to additional changes in the GCR intensity observed by neutron monitors and muon telescopes. Therefore, one may expect distortion in the temporal changes in the power-law exponent of the rigidity spectrum calculated from neutron monitor data without correcting for the changes in the cut-off rigidity of the Earth's magnetic field. We compare temporal changes in the rigidity spectrum of Fds calculated from neutron monitor data corrected and uncorrected for the geomagnetic disturbances. We show some differences in the power-law exponent of the rigidity spectrum of Fds, particularly during large disturbances of the cut-off rigidity of the Earth's magnetic field. However, the general features of the temporal changes in the rigidity spectrum of Fds remain valid as they were found in our previous study. Namely, at the initial phase of the Fd, the rigidity spectrum is relatively soft and it gradually becomes hard up to the time of the minimum level of the GCR intensity. Then during the recovery phase of the Fd, the rigidity spectrum gradually becomes soft. This confirms that the structural changes of the interplan-
\end{abstract}

M.V. Alania

Institute of Math. and Physics, Siedlce University, Siedlce, Poland

M.V. Alania

Institute of Geophysics, Tbilisi State University, Tbilisi, Georgia

A. Wawrzynczak $(\bowtie)$

Institute of Computer Science, Siedlce University, Siedlce, Poland

e-mail: awawrzynczak@uph.edu.pl

A. Wawrzynczak

National Centre for Nuclear Research, Świerk-Otwock, Poland

V.E. Sdobnov $\cdot$ M.V. Kravtsova

The Institute of Solar-Terrestrial Physics of Siberian Branch of RAS, P.O. Box 291, Irkutsk, Russia 
etary magnetic field turbulence in the range of frequencies of $10^{-6}-10^{-5} \mathrm{~Hz}$ are generally responsible for the time variations in the rigidity spectrum we found during the Fds.

Keywords Forbush decrease · Geomangetic disturbances $\cdot$ Rigidity spectrum

\section{Introduction}

The short-term depressions in the cosmic ray flux reaching the Earth are called the Forbush decreases (Fds). They are caused by the interplanetary counterpart of coronal mass ejections (CMEs) (and the shocks they drive) and also by the corotating interaction regions (CIRs) originating from the Sun.

The geomagnetic disturbances and Fds have a common origin in the interplanetary space, namely the Earth's encounter with a strong interplanetary structure. However, the magnitudes of geomagnetic disturbances and Fds are not proportional to each other (e.g., Kane, 1977, 2010). According to Dungey's (1961) mechanism, relatively high-energy particles of the solar wind rush towards the Earth but are diverted around the Earth in circular orbits in the equatorial plane. These particles form a ring current at several Earth radii and cause large geomagnetic field reduction. The reduction in the terrestrial magnetic field strength is measured by the Dst index (disturbance storm time index; Sugiura, 1964). Measurements of the Dst index and the magnitude of Fds indicate some similarity in their interplanetary sources. However, there are significant differences in the exact evolution of these indices (Cane, 2000). In general, both rise with increasing interplanetary magnetic field (IMF) and solar wind velocity $V$. The most important difference is that Fds are governed by the conditions in a large volume of the heliospheric region, while Dst variation depends on the local situation in the magnetotail near the Earth. In general not all CMEs are geoeffective, and many smaller geomagnetic disturbances are not related to CMEs.

Kudela and Brenkus (2004) analyzed the decreases in the Galactic cosmic ray (GCR) intensity along with simultaneous changes in the Dst index in the period of 1982-2002. Their analysis shows that the relation between GCR flux and geomagnetic activity is complex. The relationship between Fd magnitudes and geomagnetic activity during 1978-1996 was studied by Belov et al. (2001). By analyzing more than one thousand events they found a correlation coefficient $r \approx 0.42$ between the Fd magnitude and the maximum geomagnetic disturbance index $\mathrm{Kp}$; the correlation with Dst was smaller. They also recognized that large Fds were associated with disturbances $\mathrm{Kp} \geq 8$ in the interplanetary space causing significant changes in the Earth's magnetosphere.

Kane (2010) analyzed the relationship between the magnitude of Fds measured at the Climax station and the Dst index for $17 \mathrm{Fds}$ in the 23rd solar cycle, and showed that the maximum negative Dst generally did not occur at the time of the maximum of Fd magnitude. However, for extreme events (six events with largest Dst and corresponding Fds) in earlier cycles 19-22 he found the existence of negative correlation (correlation coefficient $r \approx-0.70$ ) between Dst and Fd magnitude. These investigations show that the relation between Fds and geomagnetic disturbances is quite complex.

One of the fundamental characteristics of Fds is the dependence of the amplitude of Fd (difference between the GCR intensity at the onset and the minimum times of an Fd) on the rigidity $R$ of GCR particles. The rigidity spectrum of an Fd can be found based on the magnitude of the Fd calculated from the minimum point of the GCR intensity. However, to study the rigidity dependence of Fd based only on the rigidity spectrum taken at one time point is not sufficient to understand its dynamics. The time evolution of the rigidity spectrum 
of the Fd was studied by Alania and Wawrzynczak $(2008,2012)$ and by Wawrzynczak and Alania (2005a, 2005b, 2010). They showed that the rigidity spectrum $\delta D(R) / D(R) \propto R^{-\gamma}$ of the great majority of Fds gradually becomes hard during the decreasing and minimum phases and then gradually becomes soft in the recovery phase. Consequently, the exponent $\gamma$ of the rigidity spectrum is large $(\gamma \approx 1-1.6)$ at the initial phase, gradually decreases up to the minimum (or near minimum) of GCR intensity ( $\gamma \approx 0.4-0.6$ ), and then increases during the recovery phase $(\gamma \approx 1-1.6)$.

Wawrzynczak and Alania (2005a, 2005b, 2010) and Alania and Wawrzynczak (2008, 2012) have shown that the temporal changes in the exponent $\gamma$ of the rigidity spectrum of the Fds found by neutron monitors and muon telescopes are related to the changes in the power spectral density (PSD) of the IMF turbulence (PSD $\propto f^{-v}$, here $f$ is the frequency). This relationship is expected owing to the dependence of the diffusion coefficient $K_{\|}$of GCR particles on the rigidity $R, K_{\|} \propto R^{\alpha}$; here the coefficient $\alpha$ depends on the exponent $v$ of the PSD of the IMF turbulence according to the quasi-linear theory (QLT) as $\alpha=2-v$ (Jokipii, 1966, 1971; Hasselman and Wibberentz, 1968; Toptygin, 1985). As was shown by Wawrzynczak and Alania (2005a, 2005b, 2010), Alania and Wawrzynczak (2008, 2012), and Alania, Iskra, and Siluszyk $(2008,2010)$, the exponent $\gamma$ is related to $\alpha$. Namely, $\gamma \approx$ $2-v$ in the range of frequency of the IMF turbulence $f \approx \sim 10^{-6}-10^{-5} \mathrm{~Hz}$, to which the neutron monitors and muon telescopes respond. The validity of the QLT (Jokipii, 1966, 1971) for the GCR particles with energy $\geq 1 \mathrm{GeV}$ is confirmed by the weakly nonlinear theory (WNLT; Shalchi et al., 2004), nonlinear parallel diffusion theory (NLPA; Qin, 2007) and by Droge (2003), Shalchi and Schlickeiser (2004), and Shalchi (2009).

All studies mentioned above are on Fds using the data from neutron monitors and muon telescopes which were uncorrected for geomagnetic disturbances associated with the Fds. However, data of neutron monitors and ground muon telescopes are generally disturbed due to changes in the vertical geomagnetic cut-off rigidity which is directly connected to the geomagnetic disturbances. Therefore, it is natural to expect that the rigidity spectrum of Fds will be distorted as well. The main purpose of this paper is threefold:

i) to calculate changes in the geomagnetic cut-off rigidity for various neutron monitor stations during three large Fds of the 23rd solar cycle (6-30 July 2000, 19 October - 11 November 2003, and 6-23 September 2005) by the spectrographic global survey method and corresponding corrections for the amplitudes of Fds,

ii) to settle the relationship between the changes in the geomagnetic cut-off rigidity and the variations in the Dst index, and then to find a reliable average relation between Dst and the correction for the GCR intensity $\Delta J$, and

iii) to estimate how the changes in the cut-off rigidity of various neutron monitor stations influence the temporal changes in the power-law exponent of the rigidity spectrum of the Fd events.

\section{Experimental Data and Analysis Methods}

We analyze the largest three Fds of the 23rd solar cycle, i.e., 6-30 July 2000 (Fd I), 19 October - 11 November 2003 (Fd II), and 6-23 September 2005 (Fd III). During these Fds were observed significant geomagnetic disturbances that could affect the vertical geomagnetic cut-off rigidity and correspondingly the level of GCR intensity registered by neutron monitors. Consequently, one could expect distortion of the rigidity spectrum of the Fds calculated from the neutron monitor data. As a result it turned out necessary to correct for the GCR intensities observed by neutron monitors by incorporating the changes in the cut-off rigidity $\Delta R_{\mathrm{c}}$ and then to calculate the rigidity spectrum of the $\mathrm{Fd}$ events. 
Figure 1 Changes in the geomagnetic cut-off rigidity $\Delta R_{\mathrm{C}}$ (left vertical axis) and the GCR intensity correction $\Delta J$ (right vertical axis) versus the geomagnetic cut-off rigidity $R_{\mathrm{C}}$ on the days of minimum of GCR intensity (daily mean) for the three considered Fds.

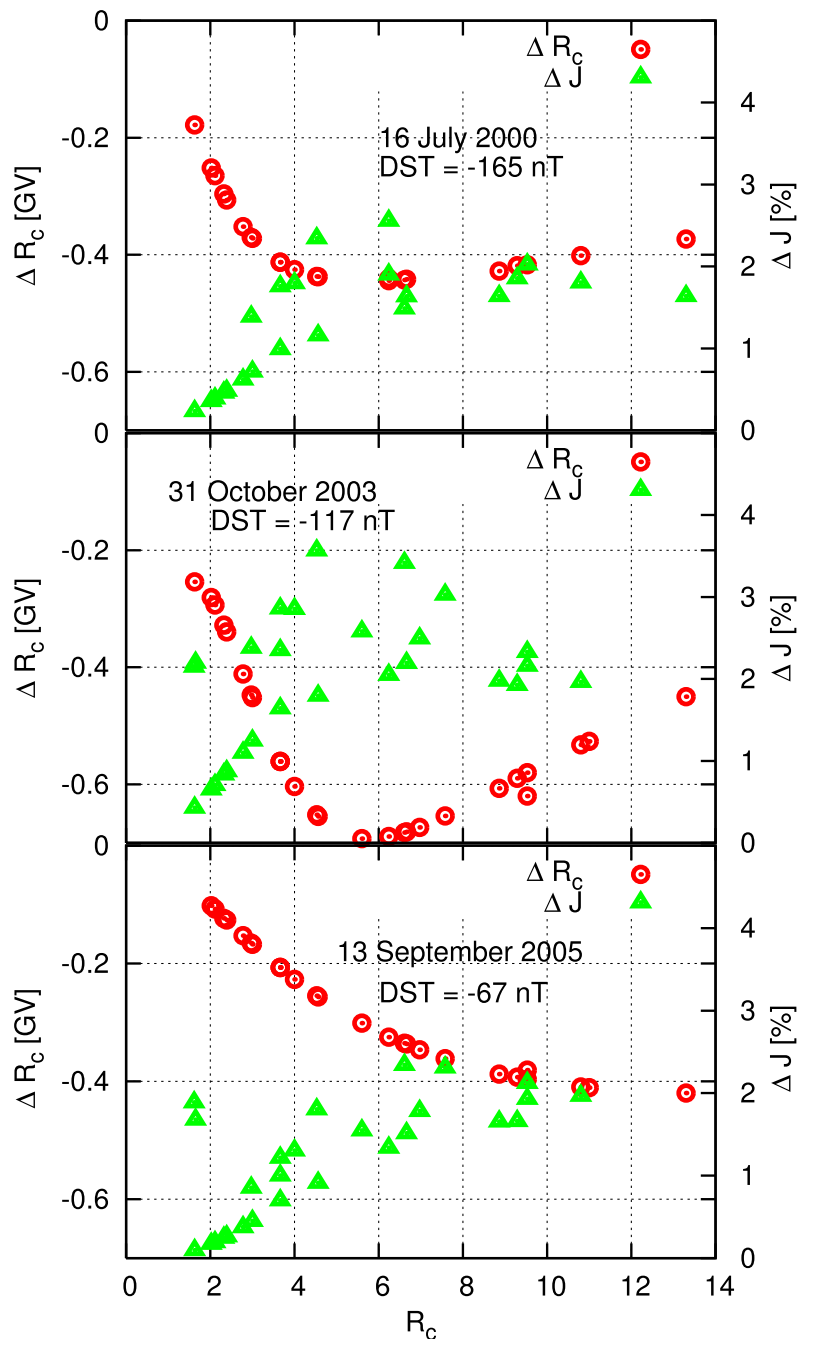

\subsection{Results from the SGS Method}

To find the changes in the cut-off rigidity $\Delta R_{\mathrm{c}}$ during the analyzed Fds from different neutron monitors we have used the spectrographic global survey (SGS) method developed by Dvornikov, Sdobnov, and Sergeev (1983) and Dvornikov and Sdobnov (2002). This method allows investigating variations in the cosmic ray rigidity spectrum and anisotropy by using the data from ground measurements, along with the changes in the geomagnetic cut-off rigidity based on the data from the world-wide network of neutron monitor stations. The list of used neutron monitor stations is given in the Appendix.

By applying the SGS method we have calculated the daily changes in $\Delta R_{\mathrm{c}}$ by using the hourly data from neutron monitor stations ( 35 stations for Fd I and 44 stations for Fd II and Fd III). The calculated values of $\Delta R_{\mathrm{c}}$ for the days of minimum intensity (i.e. 16 July 2000, 31 October 2003, and 13 September 2005) are presented by red dots in Figure 1 . The vertical axis of Figure 1 shows the changes in the cut-off rigidity $\left(\Delta R_{\mathrm{c}}\right)$, and the horizontal 
Figure 2 Changes in the Dst index (right vertical axis) and in the cut-off rigidity $\Delta R_{\mathrm{c}}$ (left vertical axis) for Climax, Hermanus, and Haleakala neutron monitor stations during the three considered Fds.

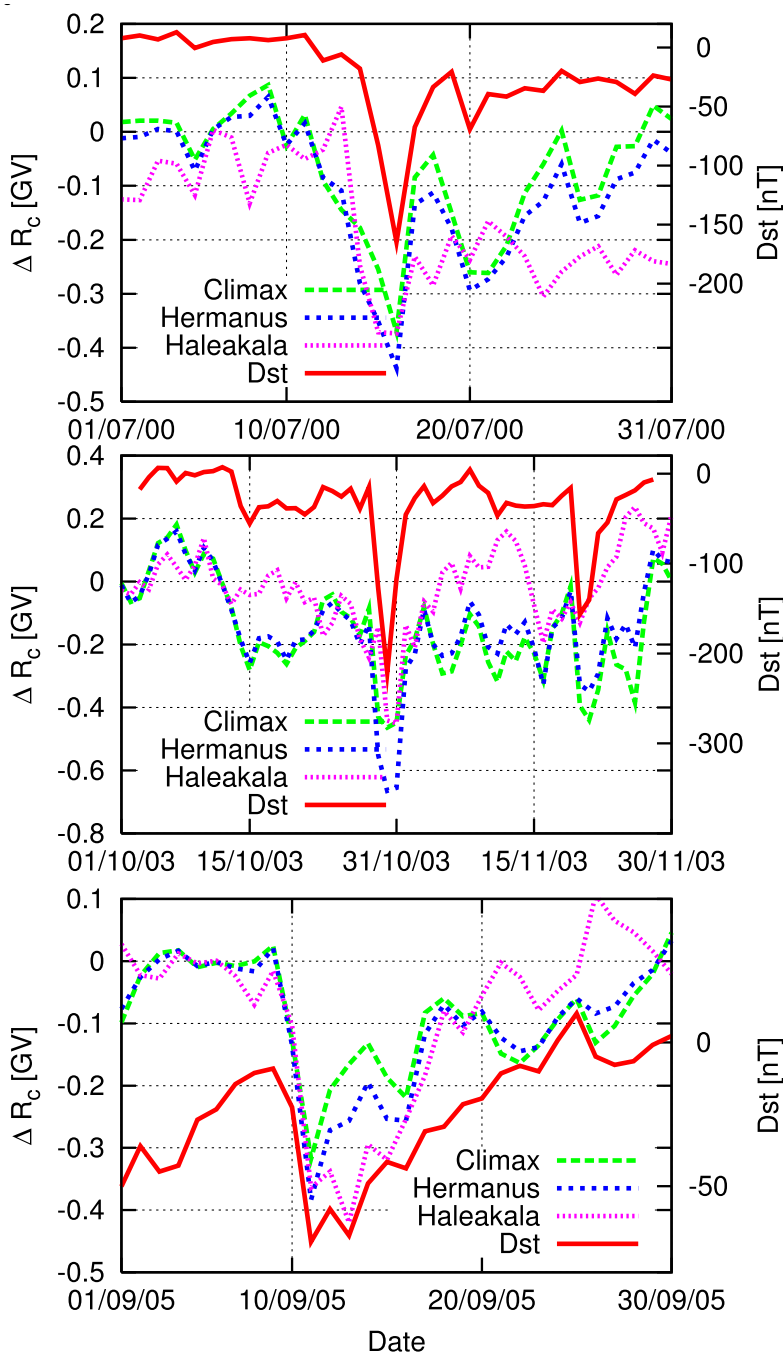

axis shows the geomagnetic cut-off rigidity $\left(R_{\mathrm{c}}\right)$ of the specified stations. Figure 1 indicates that the distributions of $\Delta R_{\mathrm{c}}$ are similar for Fd I and II with a minimum at $5-6 \mathrm{GV}$ of the cut-off rigidity. However, for Fd III no such tendencies are recognized. It is clear that each geomagnetic storm has a specific character due to a different way of interaction between the magnetosphere and ICME or flare-induced shock wave (Dessler and Parker, 1959; Perreault and Akasofu, 1978). Therefore, a complex structure of the Earth's magnetic field during magnetic storms would lead to diverse changes in $\Delta R_{\mathrm{c}}$. The corresponding corrections for the GCR intensity, $\Delta J$, caused by the changes in the geomagnetic cut-off rigidity $\Delta R_{\mathrm{c}}$ during these Fds are marked by green triangles in Figure 1 as well. Figure 1 shows very similar profiles of $\Delta J$ for all three Fds. The value of $\Delta J$ increases sharply versus $R_{\mathrm{c}}$ in the range of $2-6 \mathrm{GV}$ of the cut-off rigidity, and then gradually decreases. One can note that all Fds show significant dispersion in $\Delta J$ in the range of $R_{\mathrm{c}}=4-8 \mathrm{GV}$. The maximum changes in the GCR intensity are found in this range of $R_{\mathrm{c}}$ for all the three Fds. 
Table 1 Correlation coefficients between the changes in the Dst index and variations in the geomagnetic cut-off rigidity $\Delta R_{\mathrm{c}}$ for Climax, Hermanus, and Haleakala neutron monitor stations during three considered Fds (data presented in Figure 2).

\begin{tabular}{llll}
\hline $\begin{array}{l}\text { Correlation coefficients } \\
\text { Dst [nT] vs. } \Delta R_{\mathrm{c}}[\mathrm{GV}]\end{array}$ & $10-20$ July 2000 & 28 Oct -8 Nov 2003 & $9-25$ Sep 2005 \\
\hline Climax & $0.83 \pm 0.10$ & $0.85 \pm 0.09$ & $0.68 \pm 0.14$ \\
Hermanus & $0.85 \pm 0.09$ & $0.93 \pm 0.04$ & $0.84 \pm 0.08$ \\
Haleakala & $0.79 \pm 0.13$ & $0.77 \pm 0.13$ & $0.94 \pm 0.03$ \\
\hline
\end{tabular}

Table 2 Correlation coefficients between the changes in the Dst index and changes in the GCR intensity correction $\Delta J$ for Climax, Hermanus, and Haleakala neutron monitor stations during three considered Fds (data presented in Figure 4).

\begin{tabular}{lccc}
\hline $\begin{array}{l}\text { Correlation coefficients } \\
\text { Dst [nT] vs. } \Delta J[\%]\end{array}$ & 10-20 July 2000 & 28 Oct -8 Nov 2003 & -25 Sep 2005 \\
\hline Climax & $-0.71 \pm 0.16$ & $-0.88 \pm 0.07$ & $-0.61 \pm 0.16$ \\
Hermanus & $-0.78 \pm 0.13$ & $-0.92 \pm 0.05$ & $-0.79 \pm 0.10$ \\
Haleakala & $-0.77 \pm 0.14$ & $-0.72 \pm 0.15$ & $-0.94 \pm 0.03$ \\
\hline
\end{tabular}

There arises a natural question; what is the level of correlation between the changes in $\Delta R_{\mathrm{c}}$ and Dst monitoring the world-wide magnetic storm level, and between the changes in $\Delta J$ and Dst. The point is that $\Delta R_{\mathrm{c}}$ is calculated based on the GCR intensity changes measured by neutron monitor stations with different cut-off rigidity and should characterize an average state of the whole magnetosphere, while Dst gives information generally about the vicinity of the magnetosphere inside of the ring current, which can be extended further than 8-10 Earth's radii during geomagnetic storms (Perreault and Akasofu, 1978). The changes in the Dst index and the cut-off rigidity $\Delta R_{\mathrm{c}}$ for Climax, Hermanus, and Haleakala cosmic ray stations (low, middle, and high cut-off rigidities, correspondingly) during the three Fds are presented in Figure 2. One can note high correlation among them (Table 1). Figure 2 and Table 1 show that the minimum times of Dst and $\Delta R_{\mathrm{c}}$ coincide during each considered Fd.

It is interesting to determine the relationship between the changes in Dst and $\Delta R_{\mathrm{c}}$. For this purpose we have calculated a linear regression relation between Dst and $\Delta R_{\mathrm{c}}$ by using the data in the main phase of the three Fds. Figure 3 shows the results for six cosmic ray neutron monitor stations (crosses for July 2000, filled circles for October 2003, and squares for September 2005). The coefficients of the linear regression are given in the Appendix. In spite of large dispersion, particularly for Dst $<-(50-70) \mathrm{nT}$, one can recognize an approximately consistent dependence of $\Delta R_{\mathrm{c}}$ on Dst for Dst $>-100 \mathrm{nT}$.

One focus of our interest was to find a possible relationship between Dst and $\Delta J$. For this purpose we present in Figure 4 the changes in the Dst index and $\Delta J$ for Climax, Hermanus, and Haleakala neutron monitor stations during three Fds. Figure 4 demonstrates high correlation (Table 2) between Dst and $\Delta J$ for all neutron monitor stations during the three Fds. This gives a possibility to establish a quantitative relationship between Dst and $\Delta J$ using a linear-type regression. Figure 5 presents the regression lines between Dst and $\Delta J$ for six neutron monitor stations (crosses for July 2000, filled circles for October 2003, and squares for September 2005). The coefficients of the linear regression are given in the Appendix. 

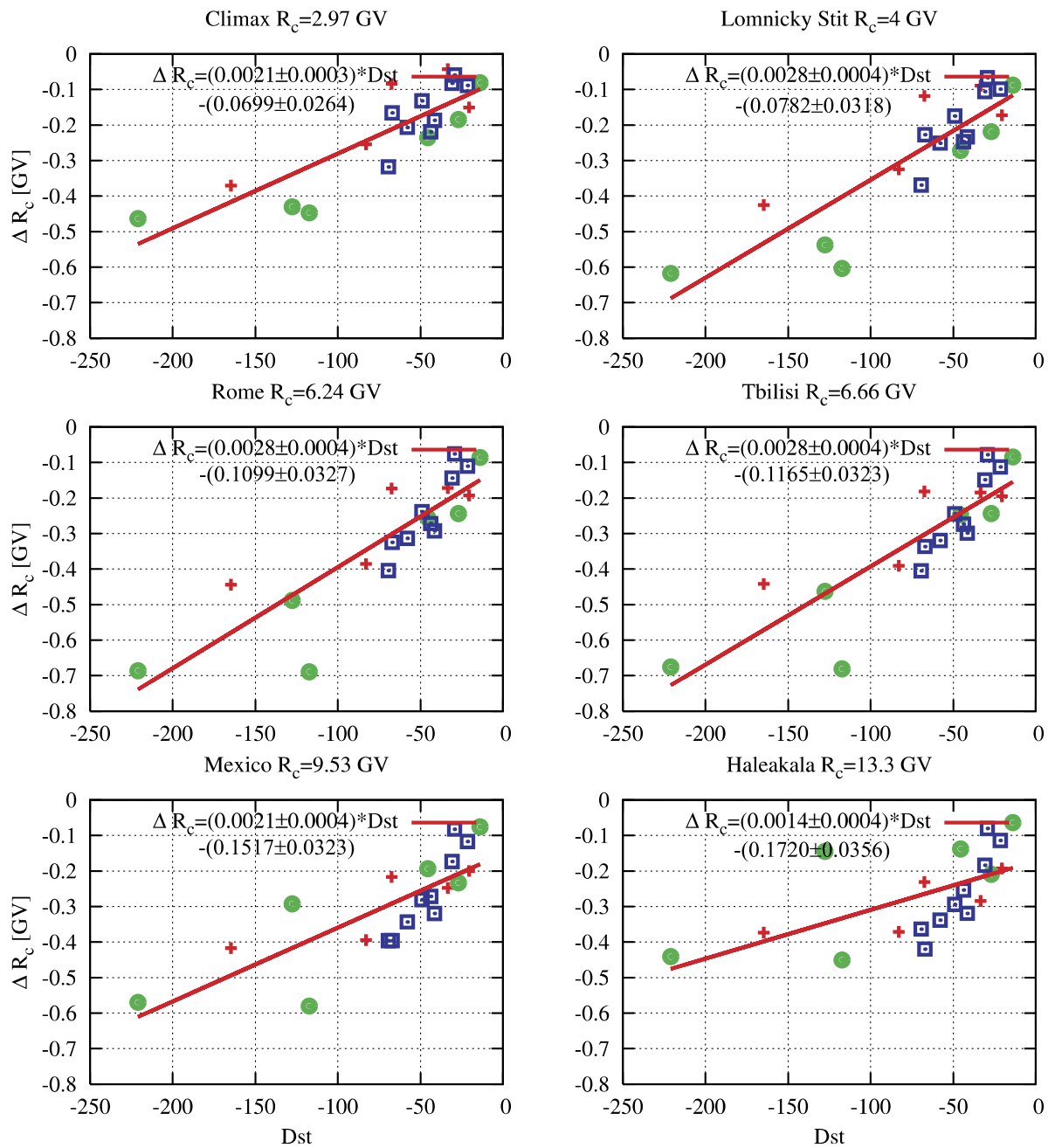

Figure 3 Relationship between the Dst index and the change in the cut-off rigidity $\Delta R_{\mathrm{c}}$ for three Fds (crosses for July 2000, filled circles for October 2003, and squares for September 2005) for neutron monitor stations with different cut-off rigidity $R_{\mathrm{c}}$. The linear regression lines are shown in red.

\subsection{Rigidity Spectrum of the Forbush Decreases}

To compare the GCR intensity for the undisturbed and disturbed magnetic fields of the Earth during the above three Fds, in Figure 6 we present the data from Climax $\left(R_{\mathrm{c}}=3.03 \mathrm{GV}\right)$, Hermanus $\left(R_{\mathrm{c}}=4.90 \mathrm{GV}\right)$, and Haleakala $\left(R_{\mathrm{c}}=13.30 \mathrm{GV}\right)$. Figure 6 shows significant changes in the GCR intensity depending on the neutron monitor stations with different cutoff rigidity for a given $\mathrm{Fd}$, and for a given neutron monitor station for different Fds. Specifically, significant differences between the corrected and uncorrected data are seen during Fd III for the Climax station and during Fd I and Fd III for the Hermanus and Haleakala stations, respectively. Generally the differences are seen around the minimum time of the GCR intensity when disturbances of the Earth's magnetic field are higher. In connection 
Figure 4 The same as Figure 2 but for the GCR intensity correction $\Delta J$ (left vertical axis).
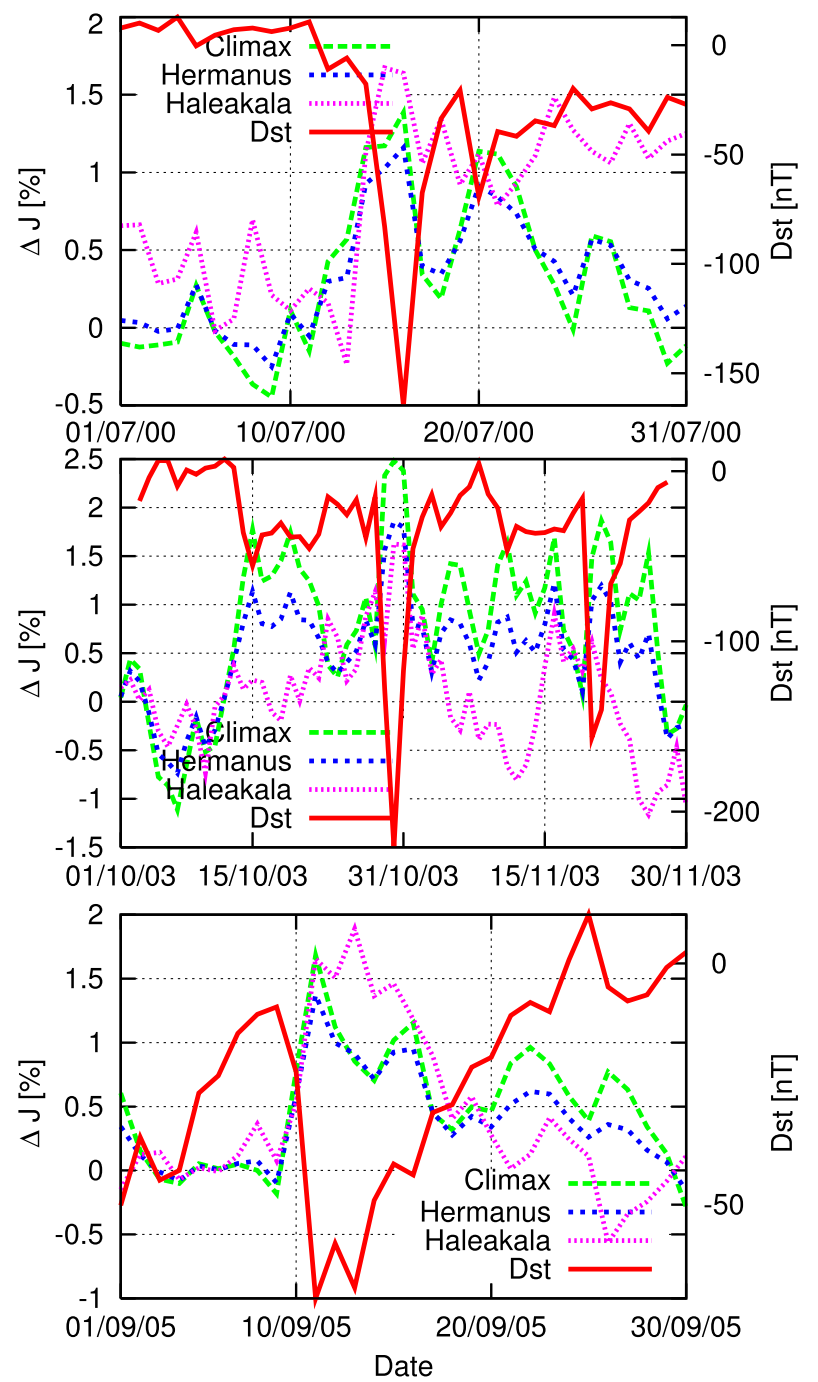

with the established differences in the GCR intensity (which we have to accept based on the valid results of the SGS method), it is interesting to know i) how the exponent $\gamma$ of the rigidity spectrum behaves in various phases of the $\mathrm{Fd}$, and ii) whether our previous findings (Wawrzynczak and Alania, 2005a, 2005b, 2008; Alania and Wawrzynczak, 2008, 2012) about the dependence of $\gamma$ on the exponent $v$ of the PSD of the IMF remain valid or not.

To answer these questions we have calculated the rigidity spectrum of the three considered Fds based on the daily measurements from neutron monitor stations smoothed over three days using both uncorrected and corrected results from the SGS method. The characteristics of the time profiles of the rigidity spectrum were analyzed for the Fd events in July 2000 by Alania and Wawrzynczak (2004), October - November 2003 by Wawrzynczak and Alania (2005a, 2005b), and September 2005 by Wawrzynczak and Alania (2010) and Alania and Wawrzynczak (2012). In this paper we focus on the comparison between the 

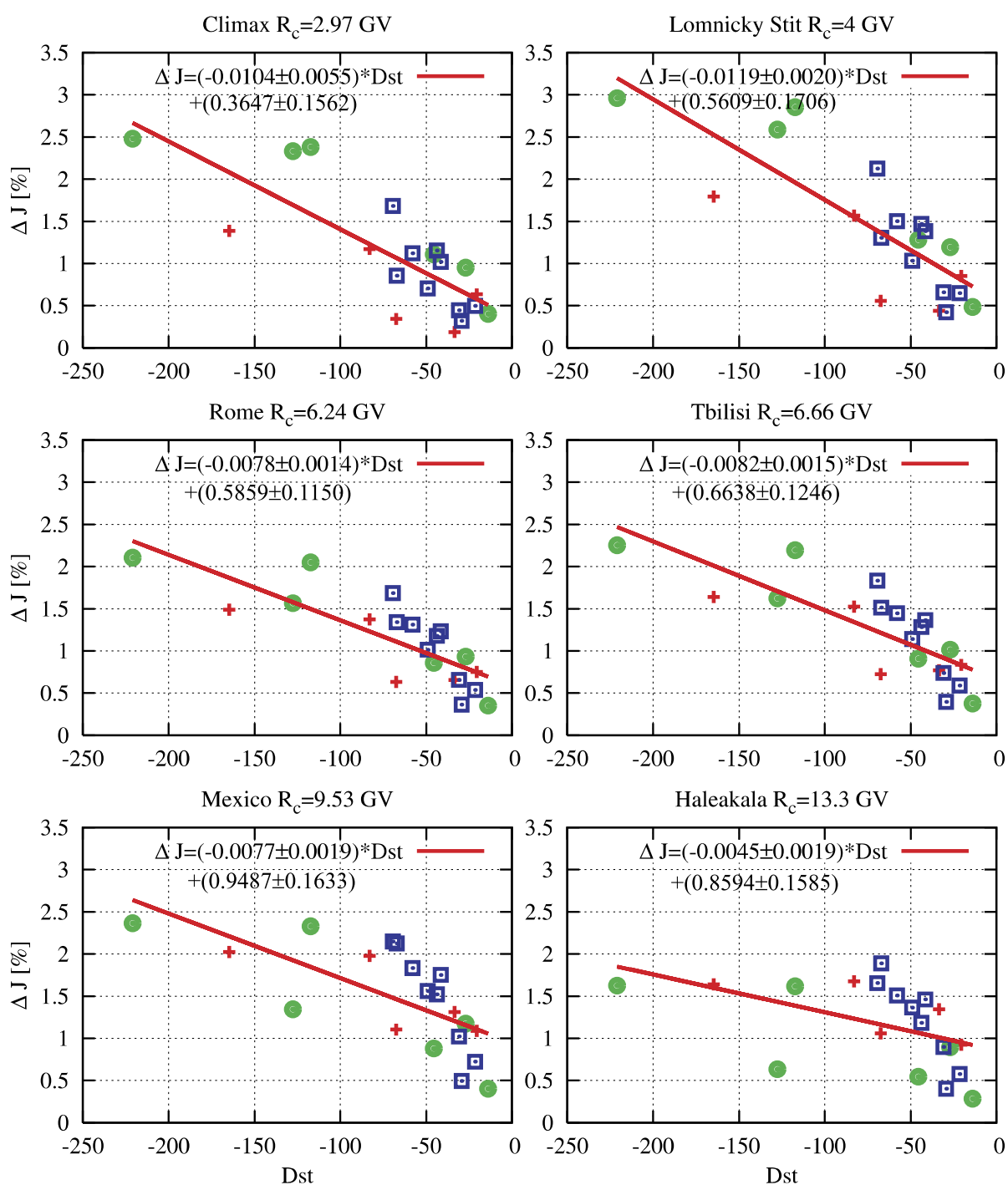

Figure 5 The same as Figure 3 but for the GCR intensity correction $\Delta J$.

rigidity spectra calculated from the neutron monitor station data which are uncorrected and corrected for the geomagnetic disturbances. The exponent $\gamma$ of the rigidity spectrum was calculated for each day of the Fd based on the data uncorrected and corrected for the cut-off rigidity changes. Amplitudes of the Fd were calculated with respect to the average intensity during three days before the start of the Fd. Based on the method described in detail in Wawrzynczak and Alania (2010) and in Alania and Wawrzynczak (2012), we have calculated the exponent $\gamma$ of the GCR variations which can be written (i.e. Dorman, 1963, 2004; Ahluwalia and Ericksen, 1971) as follows:

$$
\frac{\delta D(R)}{D(R)}= \begin{cases}A\left(\frac{R}{R_{0}}\right)^{-\gamma} & \text { for } R \leq R_{\max }, \\ 0 & \text { for } R>R_{\max },\end{cases}
$$


Figure 6 The amplitudes of the GCR intensity $J$ (left vertical axis) corrected (subscript c) and uncorrected (subscript $u$ ) for the cut-off rigidity variation for Haleakala (Ha), Hermanus (He), and $\mathrm{Climax}(\mathrm{Cl})$ neutron monitor stations during the three considered Fds. The corresponding changes in the Dst index (right vertical axis) are also shown.
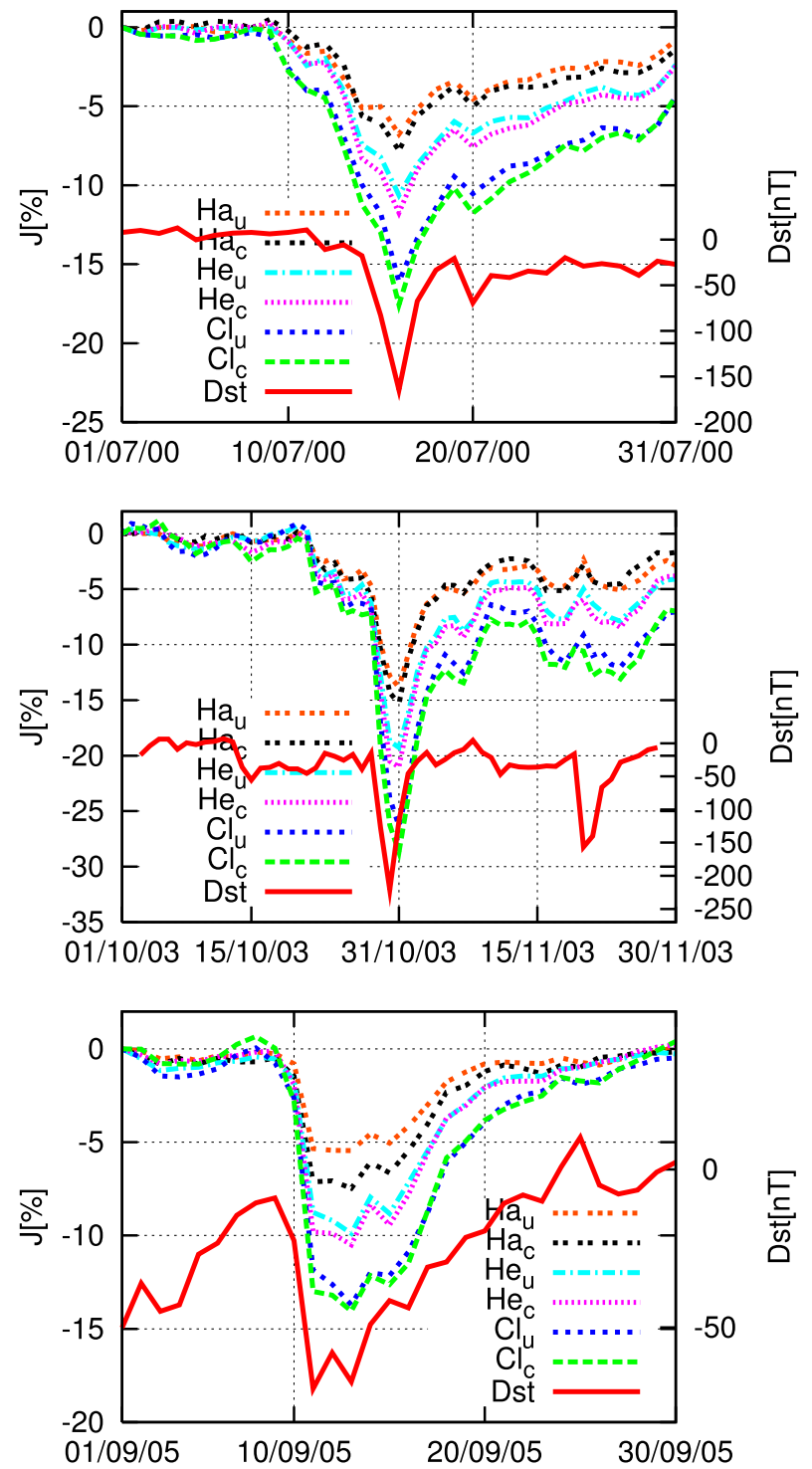

where $R_{\max }$ is the rigidity beyond which the Fd of GCR intensity is not observed and $A$ is a constant equal to the value of $\frac{\delta D(R)}{D(R)}$ for rigidity $R=R_{0}$.

Figures 7-9 present the temporal changes in the exponent $\gamma$ calculated from the uncorrected and corrected data of the Fd in July 2000 (Figure 7), October-November 2003 (Figure 8), and September 2005 (Figure 9). One can find that there are some differences between the values of the exponent $\gamma$, particularly during large disturbances in the cut-off rigidity of the Earth's magnetic field. Generally, the rigidity spectrum obtained from the corrected data is harder than that obtained from the uncorrected data. We ascribe it to unexpected large changes in the cut-off rigidity in the range of 5-8 GV. We must stress, however, that the general features of the temporal changes in the rigidity spectrum of the Fd remain as 


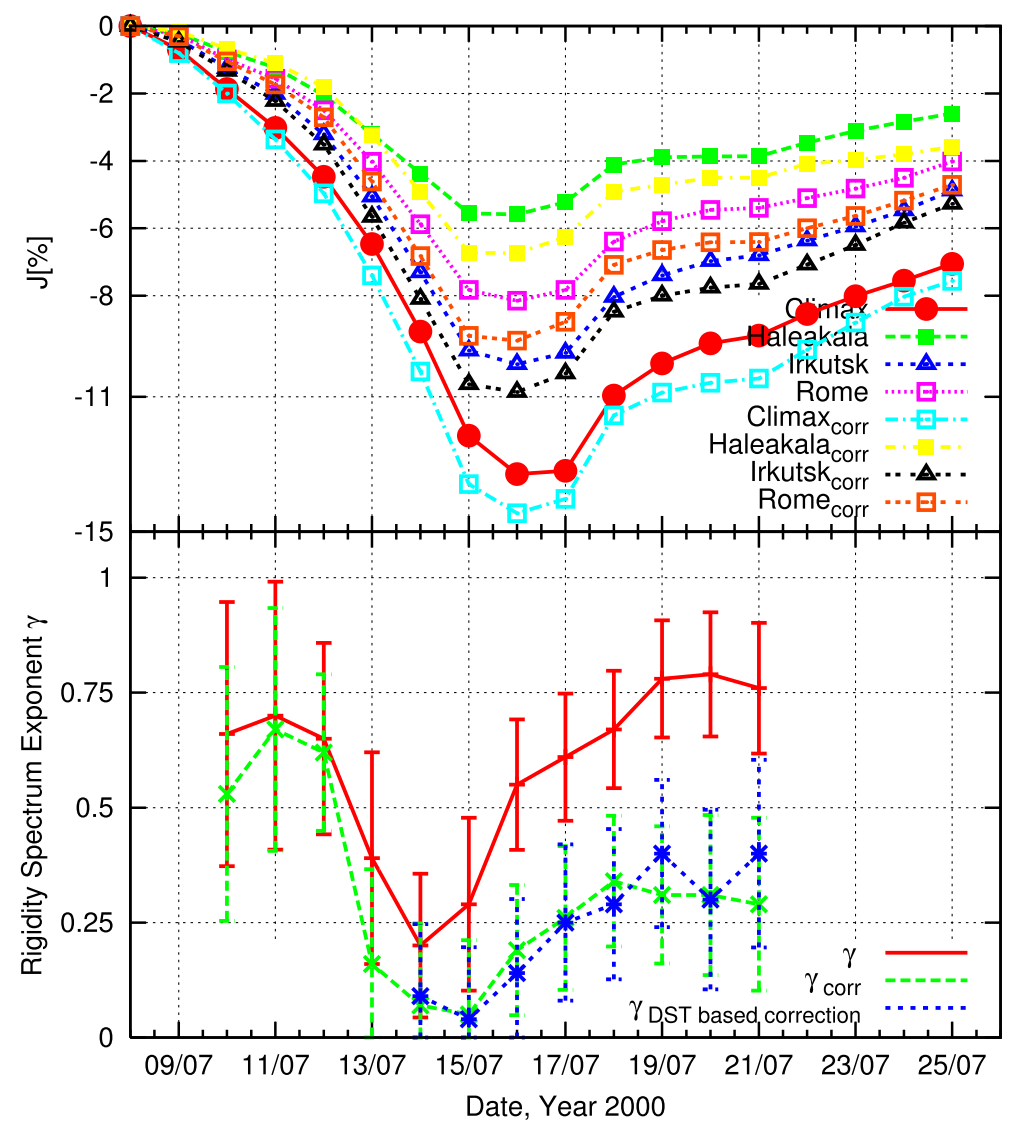

Figure 7 Temporal changes in the corrected and uncorrected GCR intensity (top panel) and in the rigidity spectrum exponent $\gamma$ (bottom panel) calculated from the corrected and uncorrected data for the period of $8-25$ July 2000.

they were found in our previous investigations (i.e. Wawrzynczak and Alania, 2005a, 2005b, 2010). Namely, at the initial phase of the Fd, the rigidity spectrum is relatively soft and it gradually hardens up to the time of the minimum level of the GCR intensity. Then during the recovery phase of the Fd the rigidity spectrum gradually softens. The same time profile of the exponent $\gamma$ supports that the structural changes in the IMF turbulence in the range of frequencies of $10^{-6}-10^{-5} \mathrm{~Hz}$, to which the neutron monitors respond, are mainly responsible for the time variations in the rigidity spectrum in the Fd (Wawrzynczak and Alania, 2005a, 2005b, 2010).

Figures 7-9 also present the rigidity spectrum exponent $\gamma$ of Fds calculated from the amplitudes of the Fds corrected for the geomagnetic disturbances by the obtained relation between the Dst index and the correction for the GCR intensity $\Delta J$ (see the Appendix). The data from the neutron monitor stations used in the calculation were corrected on the days when Dst index was $<-30 \mathrm{nT}$. One can see (Figures $7-9$ ) that the rigidity spectrum exponent $\gamma$ obtained this way (the blue line) fits within the error bars the exponent $\gamma$ calculated from the GCR intensity corrected by the result of the SGS method. We infer that the relation 


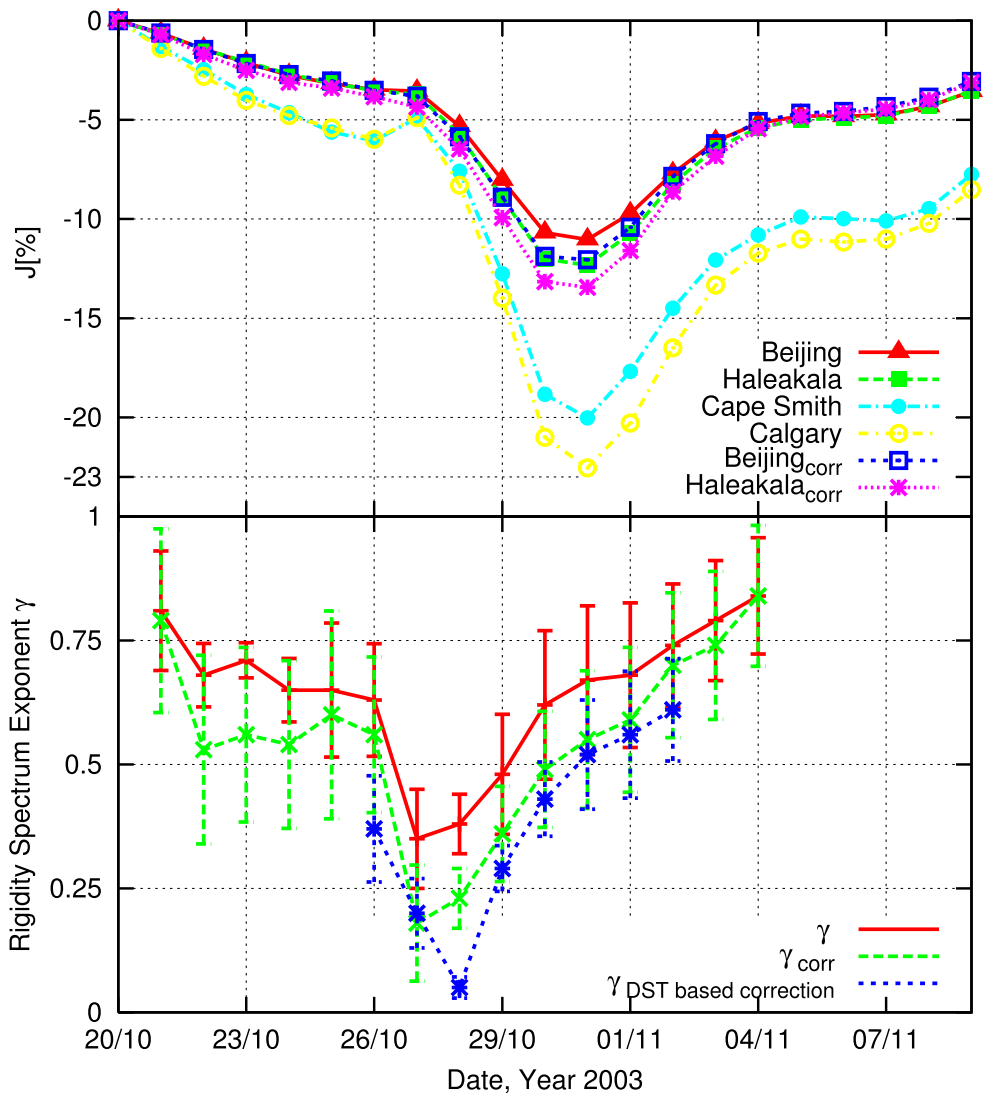

Figure 8 The same as Figure 7 but for the period of 20 October -9 November 2003.

between Dst and $\Delta J$ can be used for the estimation of the rigidity spectrum exponent $\gamma$ taking into account the geomagnetic disturbances.

\section{Summary and Conclusions}

The spectrographic global survey (SGS) method was applied to calculate the changes in the vertical cut-off rigidity caused by geomagnetic disturbances during three large Fds of the 23rd solar cycle (6-30 July 2000, 19 October - 11 November 2003, and 6-23 September 2005). The data from the neutron monitor stations were corrected for geomagnetic disturbances during the considered three Fds. The power law exponent $\gamma$ of the rigidity spectrum $\left(\delta D(R) / D(R) \propto R^{-\gamma}\right)$ of Fds found by neutron monitor station data uncorrected for geomagnetic disturbances is different from that corrected for them. Namely, the rigidity spectrum obtained from the corrected data is harder than that calculated from the uncorrected data. However, the time profiles of the changes in the exponent $\gamma$ in both cases are similar. At the initial phase of the Fd, the rigidity spectrum is relatively soft ( $\gamma$ is large) and it gradually becomes hard ( $\gamma$ decreases) up to the time of the (near) minimum level of the GCR intensity. Then during the recovery phase the rigidity spectrum gradually becomes soft ( $\gamma$ increases). This supports the previously presented results (i.e. Alania and Wawrzynczak, 


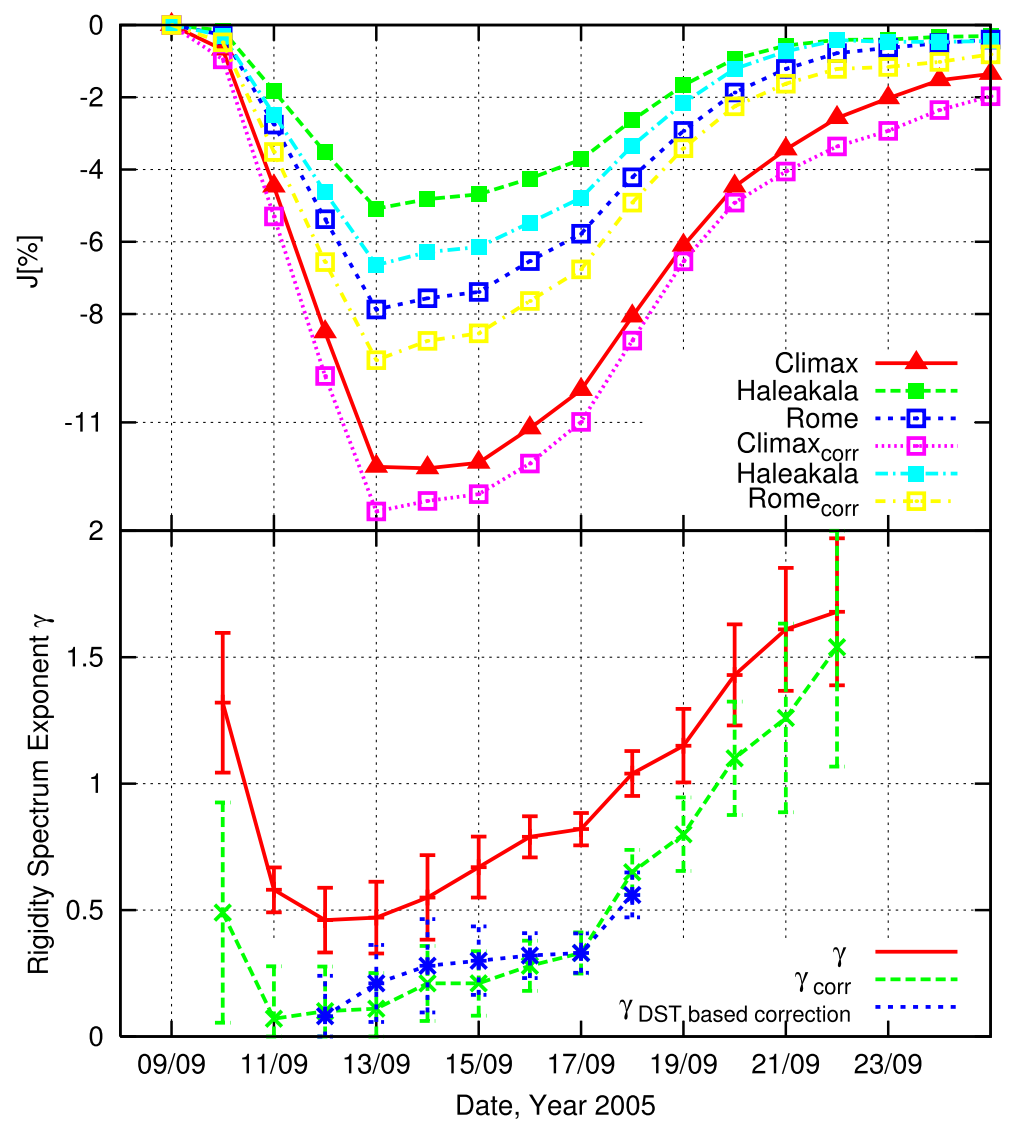

Figure 9 The same as Figure 7 but for the period of 9-23 September 2005.

2008, 2012), vindicating that the structural changes in the IMF turbulence in the range of frequencies of $10^{-6}-10^{-5} \mathrm{~Hz}$, to which the neutron monitors and muon telescopes respond, are mainly responsible for the time variations in the exponent $\gamma$ of the rigidity spectrum of Fds. The presented calculations show that, to an acceptable extent, the data from neutron monitor stations can be corrected for geomagnetic disturbances during Fds using the Dst index.

Open Access This article is distributed under the terms of the Creative Commons Attribution License which permits any use, distribution, and reproduction in any medium, provided the original author(s) and the source are credited.

\section{Appendix}

The neutron monitor stations used in the calculations of the SGS method are listed in Table 3. Parameters listed are the coefficients of the regression relation between the Dst index $[\mathrm{nT}]$ and the change in the cut-off rigidity $\Delta R_{\mathrm{c}}[\mathrm{GV}]$, and between Dst and the intensity correction $\Delta J[\%]$. The changes in the cut-off rigidity for neutron monitor stations with cut-off rigidity $<2 \mathrm{GV}$ are neglected. 


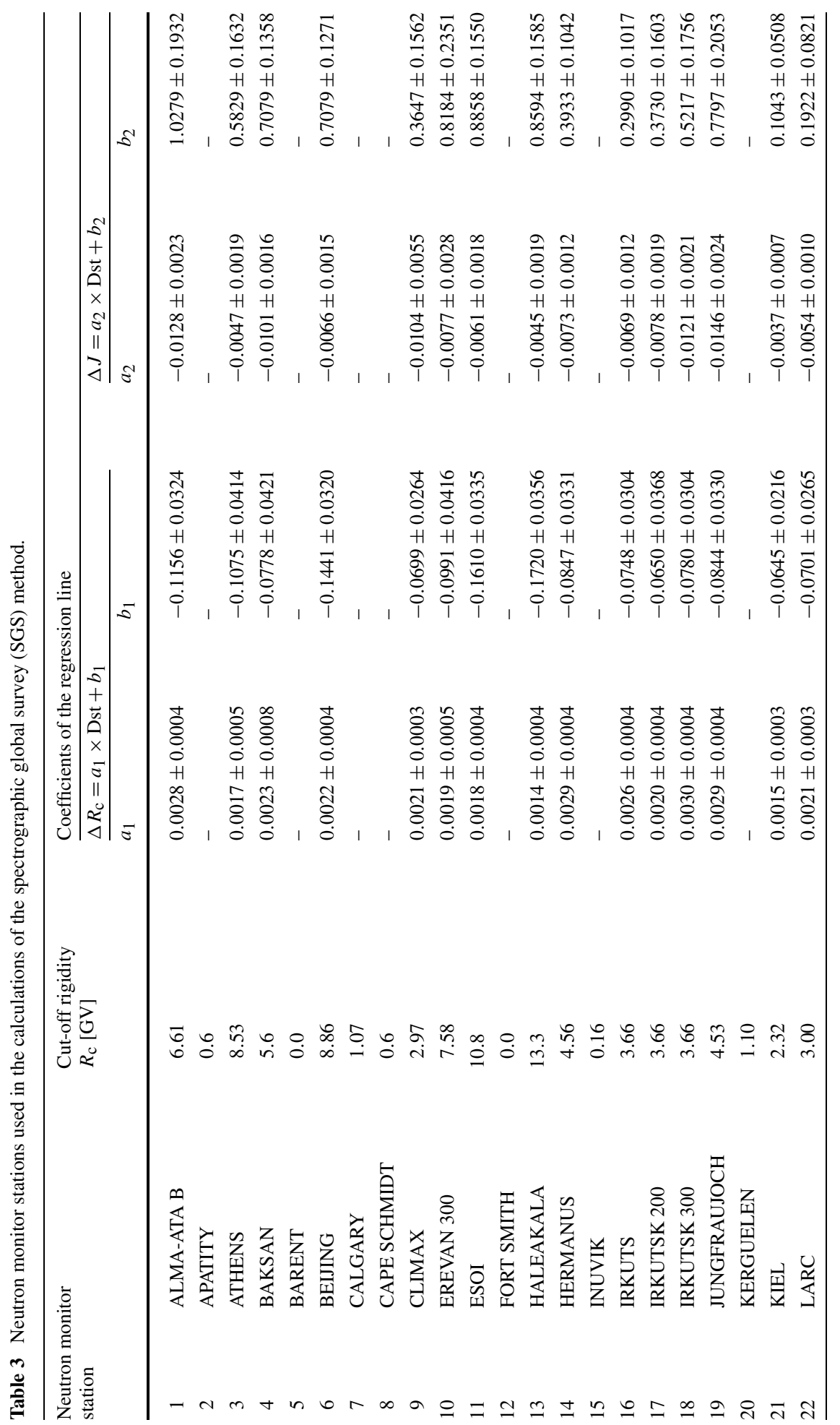




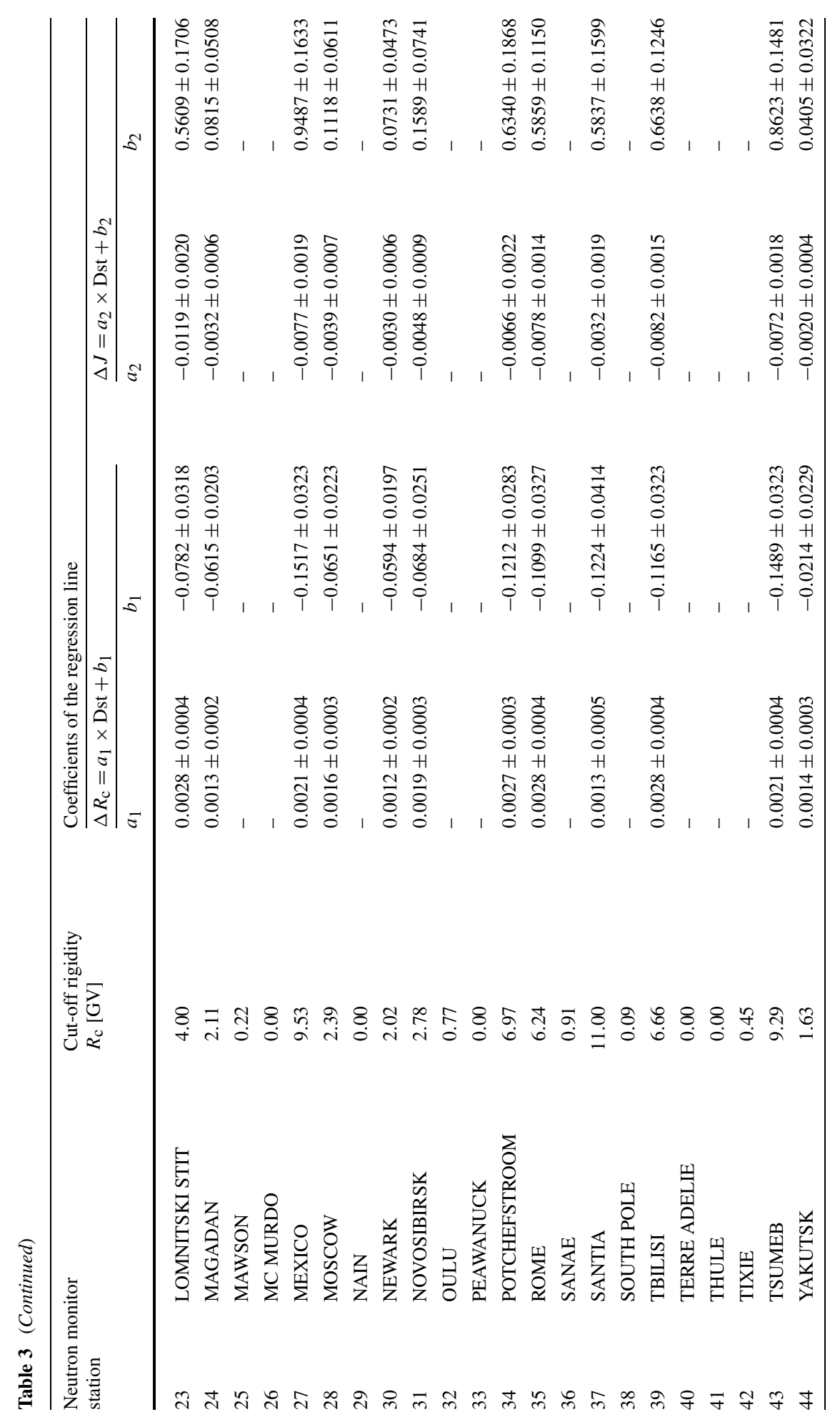




\section{References}

Ahluwalia, H.S., Ericksen, J.H.: 1971, J. Geophys. Res. 76, 6613.

Alania, M.V., Wawrzynczak, A.: 2004, Acta Phys. Pol. B 35, 1551.

Alania, M.V., Wawrzynczak, A.: 2008, Astrophys. Space Sci. Trans. 4, 59.

Alania, M.V., Wawrzynczak, A.: 2012, Adv. Space Res. 50, 725.

Alania, M.V., Iskra, K., Siluszyk, M.: 2008, Adv. Space Res. 41, 267.

Alania, M.V., Iskra, K., Siluszyk, M.: 2010, Adv. Space Res. 45, 1203.

Belov, A.V., Eroshenko, E.A., Oleneva, V.A., Struminsky, A.B., Yanke, V.G.: 2001, Adv. Space Res. $27,625$.

Cane, H.V.: 2000, Space Sci. Rev. 93, 55.

Dessler, A.J., Parker, E.N.: 1959, J. Geophys. Res. 64, 2239.

Dorman, L.I.: 1963, Cosmic Rays Variations and Space Exploration, Nauka, Moscow, 197.

Dorman, L.I.: 2004, Cosmic Rays in the Earth's Atmosphere and Underground, Kluwer Academic, Dordrecht, 140.

Droge, W.: 2003, Astrophys. J. 589, 1027.

Dungey, J.W.: 1961, Phys. Rev. Lett. 6, 47.

Dvornikov, V.M., Sdobnov, V.E., Sergeev, A.V.: 1983 In: Proc. 18th Int. Cosmic Ray Conf. 3, 249.

Dvornikov, V.M., Sdobnov, V.E.: 2002, Int. J. Geomagn. Aeron. 3, 217.

Hasselman, K., Wibberentz, G.: 1968, Z. Geophys. 34, 353.

Jokipii, J.R.: 1966, Astrophys. J. 146, 480.

Jokipii, J.R.: 1971, Rev. Geophys. Space Phys. 9, 27.

Kane, R.P.: 1977, J. Geophys. Res. 82, 561.

Kane, R.P.: 2010, Ann. Geophys. 28, 479.

Kudela, K., Brenkus, R.: 2004, J. Atmos. Solar-Terr. Phys. 66, 1121.

Qin, G.: 2007, Astrophys. J. 656, 217.

Perreault, P., Akasofu, S.-I.: 1978, Geophys. J. Roy. Astron. Soc. 54, 547.

Shalchi, A.: 2009, Nonlinear Cosmic Ray Diffusion Theories, Springer, Berlin, 180.

Shalchi, A., Bieber, J.W., Matthaeus, W.H., Qin, G.: 2004, Astrophys. J. 616, 617.

Shalchi, A., Schlickeiser, R.: 2004, Astrophys. J. 604, 861.

Sugiura, M.: 1964, Ann. Int. Geophys. Year 35, 945.

Toptygin, I.N.: 1985, Cosmic Rays in Interplanetary Magnetic Fields, Reidel Publishing Company, Dordrecht, 81.

Wawrzynczak, A., Alania, M.V.: 2005a, Acta Phys. Pol. B 36, 1847.

Wawrzynczak, A., Alania, M.V.: 2005b, Adv. Space Res. 35, 682.

Wawrzynczak, A., Alania, M.V.: 2008, Adv. Space Res. 41, 325.

Wawrzynczak, A., Alania, M.V.: 2010, Adv. Space Res. 45, 622. 